\title{
Case Report \\ Genetic Profiling of Malignant Melanoma Arising from an Ovarian Mature Cystic Teratoma: A Case Report
}

\author{
Kohei Nakamura ${ }^{1,2, *}$, Eriko Aimono ${ }^{1}$, Reika Takamatsu ${ }^{1}$, Shigeki Tanishima ${ }^{3}$, Tomonari Tohyama ${ }^{4}$, \\ Katsutoshi Sasano ${ }^{4}$, Hiroshi Sakuma ${ }^{2}$ and Hiroshi Nishihara ${ }^{1}$ \\ 1 Genomics Unit, Keio Cancer Center, Keio University School of Medicine, 35 Shinanomachi, Shinjukuku, \\ Tokyo 160-8582, Japan; eriko0123@keio.jp (E.A.); reika-t@keio.jp (R.T.); hnishihara1971@keio.jp (H.N.) \\ 2 Department of Obstetrics and Gynecology, Kumagaya General Hospital, Saitama 360-8657, Japan; \\ h-sakuma@kumasou.or.jp \\ 3 Department of Biomedical Informatics, Kansai Division, Mitsubishi Space Software Co., Ltd., Tokyo 105-6132, \\ Japan; Tanishima.Shigeki@mss.co.jp \\ 4 Department of Laboratory, Kumagaya General Hospital, Saitama 360-8657, Japan; \\ k-byouri@kumasou.or.jp (T.T.); k-sasano@kumasou.or.jp (K.S.) \\ * Correspondence: knakamura320@keio.jp; Tel.: +81-3-5315-4374
}

Citation: Nakamura, K.; Aimono, E.; Takamatsu, R.; Tanishima, S.;

Tohyama, T.; Sasano, K.; Sakuma, H.; Nishihara, H. Genetic Profiling of Malignant Melanoma Arising from an Ovarian Mature Cystic Teratoma: A Case Report. Int. J. Mol. Sci. 2021, 22, 2436. https://doi.org/10.3390/ ijms22052436

Academic Editor: Eric Vallender

Received: 30 January 2021

Accepted: 26 February 2021

Published: 28 February 2021

Publisher's Note: MDPI stays neutral with regard to jurisdictional claims in published maps and institutional affiliations.

Copyright: (C) 2021 by the authors Licensee MDPI, Basel, Switzerland This article is an open access article distributed under the terms and conditions of the Creative Commons Attribution (CC BY) license (https:// creativecommons.org/licenses/by/ $4.0 /)$.

\begin{abstract}
Ovarian mature cystic teratomas comprise tissues derived from all three germ layers. In rare cases, malignant tumors arise from ovarian mature cystic teratoma. A variety of tumors can arise from mature cystic teratoma, among which primary malignant melanoma (MM), for which no molecular analyses such as genomic sequencing have been reported to date, is exceedingly rare, thereby limiting possible therapeutic options using precision medicine. We used targeted gene sequencing to analyze the status of 160 cancer-related genes in a patient with $\mathrm{MM}$ arising from an ovarian mature cystic teratoma (MM-MCT). KRAS amplification and homozygous deletion in PTEN and RB1 were detected in tumor samples collected from the patient. No KRAS amplification has been previously reported in cutaneous MM, indicating that the carcinogenesis of MM-MCT differs from that of primary cutaneous melanomas. A better understanding of the underlying genetic mechanisms will help clarify the carcinogenesis of MM-MCT. In turn, this will enable treatment with novel targeting agents as well as the initial exploration of gene-based precision oncological therapies, which aim to improve treatment outcomes for patients with this disease.
\end{abstract}

Keywords: malignant melanoma; mature cystic teratoma; genome sequencing; precision medicine

\section{Introduction}

Malignant melanoma (MM) is the most common primary cutaneous melanoma (PCM), and its molecular and pathological characteristics have been well investigated [1,2]. The key cellular pathways, such as CDKN2A/CDK4/CCND1/RB1, MAPK, and PI3K/AKT, and significant genes, such as BRAF, NRAS, TERT, TP53, NF1, and RB1, are considered to play critical roles in PCM tumorigenesis [3-6].

$\mathrm{MM}$ arising from an ovarian mature cystic teratoma (MCT) (MM-MCT) is extremely rare. To our knowledge, fewer than 40 cases of MM-MCT have been reported to date since the first case was reported in 1901 by Andrews [7-10], and there have been no reports on its genetic profile. The diagnosis of MM-MCT prior to surgery is impossible. Furthermore, its etiopathogenesis and predictive factors are not understood, and effective treatment methods for such tumors remain elusive due to their paucity.

Precision medicine, using approaches such as comprehensive genome sequencing, is a possible treatment option for several cancers. In particular, recently developed molecular and whole-exome sequencing is being used to reveal the genetic basis of MM-MCT [11].

In the present study, we analyzed 160 cancer-related genes from a patient with MMMCT. This information will help clarify the carcinogenesis of MM-MCT and will be useful in identifying potential therapeutic targets for future precision medicine approaches. 


\section{Case Presentation}

The patient was a 62-year-old woman who underwent right salpingo-oophorectomy based on the preoperative diagnosis of an ovarian MCT. A preoperative laboratory examination showed elevated serum levels of cancer antigen (CA) 19-9 and CA125 (10,159 and $62 \mathrm{U} / \mathrm{mL}$, respectively). There was no family history.

Macroscopically, the ovarian tumor had formed a small sac in a huge cyst that consisted of fibrotic walls and dark brown contents. Small, pigmented nodules were scattered in the stroma of the walls (Figure 1). A microscopic examination revealed that the huge cyst was lined with hemosiderin-laden or foamy macrophages, and there was no lining of epithelial cells (Figure 2). A small sac was lined with squamous-type epithelium and respiratory epithelium (Figure 3). Scattered pigmented nodules (maximum of $14 \mathrm{~mm}$ ) were composed of the medullary proliferation of anaplastic cells with enlarged nuclei (Figure $4 a, b)$. The immunohistochemical staining of atypical cells revealed a positive result for MelanA (Figure 4c) and HMB45. In addition, high power field observation confirmed the presence of melanin granules that were positive for Fontana-Masson stain (Figure 4d) and negative for colloidal iron stain (Figure 4e). Based on these observations, we made the primary diagnosis of MM accompanied with an MCT and an endometriotic cyst. The gross or microscopic findings alone could not indicate the origin of the melanoma derived from the MCT or endometriotic cyst.
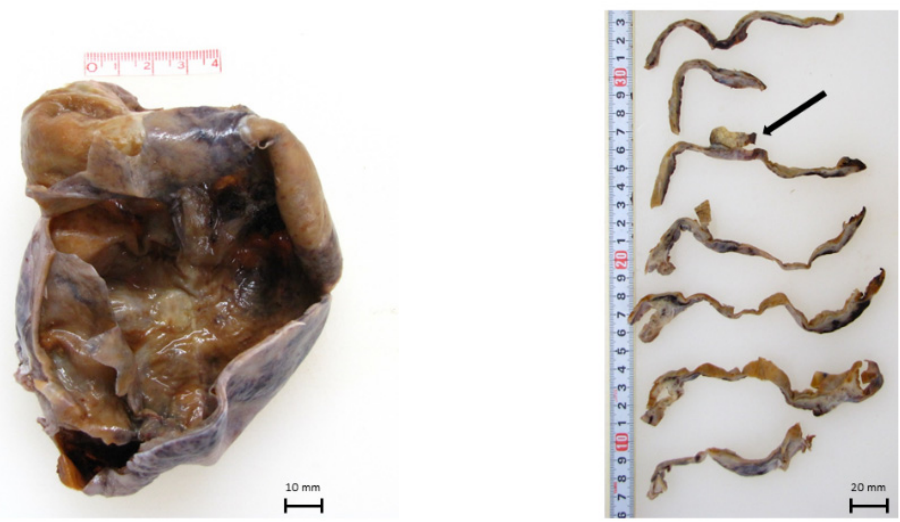

Figure 1. Macroscopic findings: The tumor comprised a small sac (arrow) in a huge cystic legion.

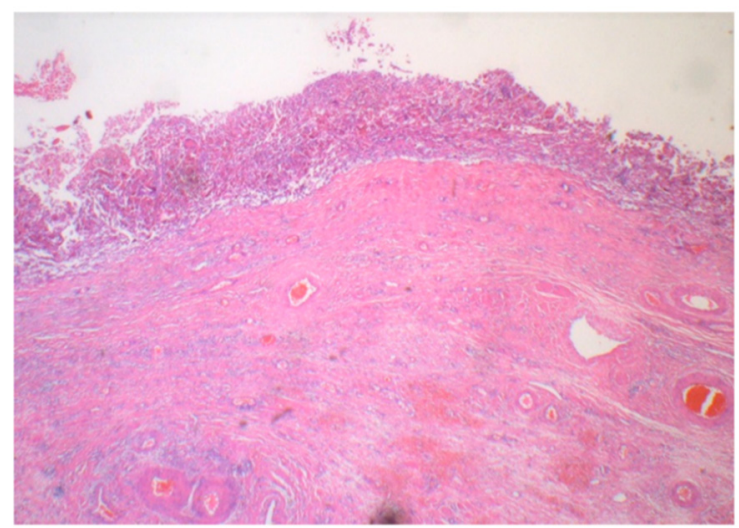

Figure 2. Histologically, the huge cyst was lined with hemosiderin-laden macrophages, and the epithelium was exfoliated (H\&E staining) $(5 \times)$. 


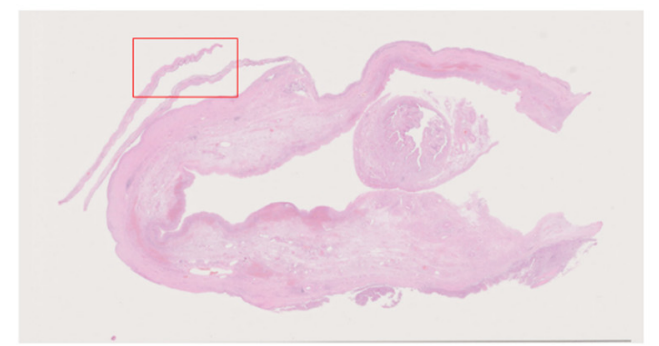

(a)

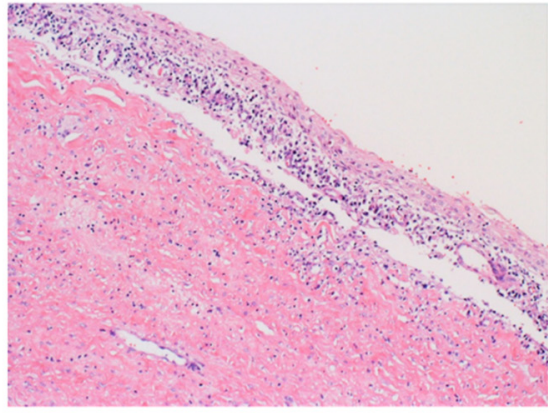

(b)

Figure 3. A mature cystic teratoma was observed in a small sac, containing squamous epithelium (low-power: (a: $0.4 \times)$, high-power: $(\mathbf{b}: 5 \times)$ ) (H\&E staining).

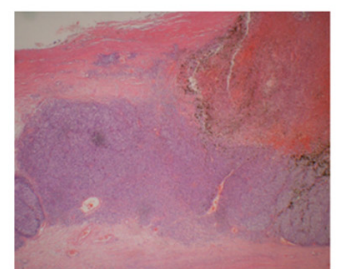

(a)

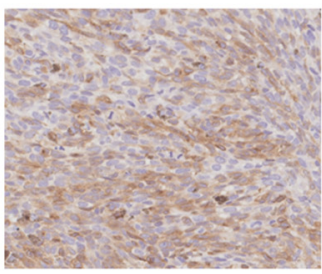

(c)

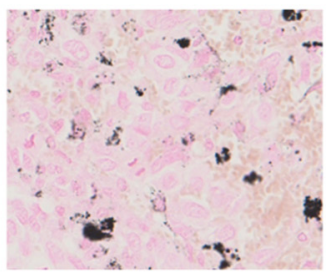

(d)

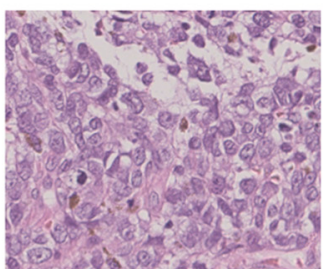

(b)

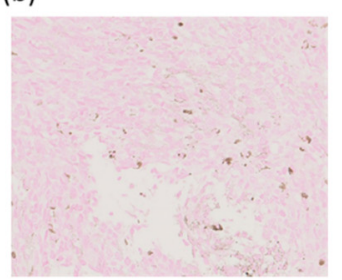

(e)

Figure 4. Fibrotic wall showing scattered pigmented areas (a: $2 \times)$. Tumor cells consisted of enlarged nuclei and melanin granules (b: $20 \times$ ). Immunohistochemically, tumor cells were positive for MelanA $($ c: $10 \times)$. The granules were positive for Fontana-Masson $(\mathbf{d}: 20 \times)$, and negative for collioid iron staining $(\mathbf{e}: 10 \times)$.

After the surgery, we performed a PET-CT scan and confirmed the diagnosis as MMMCT. The patient underwent pembrolizumab treatment, which is a standard treatment for PCM. However, the liver metastasis progressed, and the treatment was not effective. Subsequent ipilimumab and radiation therapy to the liver metastasis ( $20 \mathrm{~Gy} / 5 \mathrm{fr})$ also failed to control the disease. Two years after the surgery, the patient died of progressive disease.

Genomic DNA was obtained from a sample classified as MM-MCT, but not from mature cystic teratoma and endometriotic cyst, which was thought to be a separate precursor lesion because of the shortage of the sample volume. We performed a cancer gene profiling test by PleSSision-160, as previously described [12-14]. The average sequencing depth was $706.5 \times$ for MM-MCT. The average tumor cellularity was $70 \%$, as determined histologically.

Several actionable gene alterations were detected in the MM-MCT sample as follows. Homozygous deletion (HD) was detected in both PTEN and RB1 (Figure 5). Oncogene amplification was detected in KRAS (estimated copy number: 4.4) (Figure 5). TSC1 (p.P366Q) and EPCAM (p.L286Afs*13) variants were considered as variants of unknown significance. The tumor cells of MM-MCT showed a lack of immunoreactivity for PTEN and RB1, which is consistent with PTEN/RB1 HD status. After analyzing DNA, we examined the results of immunohistochemical staining. The tumor cells of MM were negative for PTEN and RB1 (Figure 6a,b), which is consistent with PTEN/RB1 HD status. Conversely, the epithelium of the MCT showed a mosaic pattern for PTEN and RB1 staining (Figure 7a,b). Regarding the endometriosis lesions, we could not evaluate the expression of PTEN and RB1 as there 
were no epithelial cells. However, we concluded that the MM had probably arisen from MCT because PTEN and RB1 HD status was observed in both lesions.
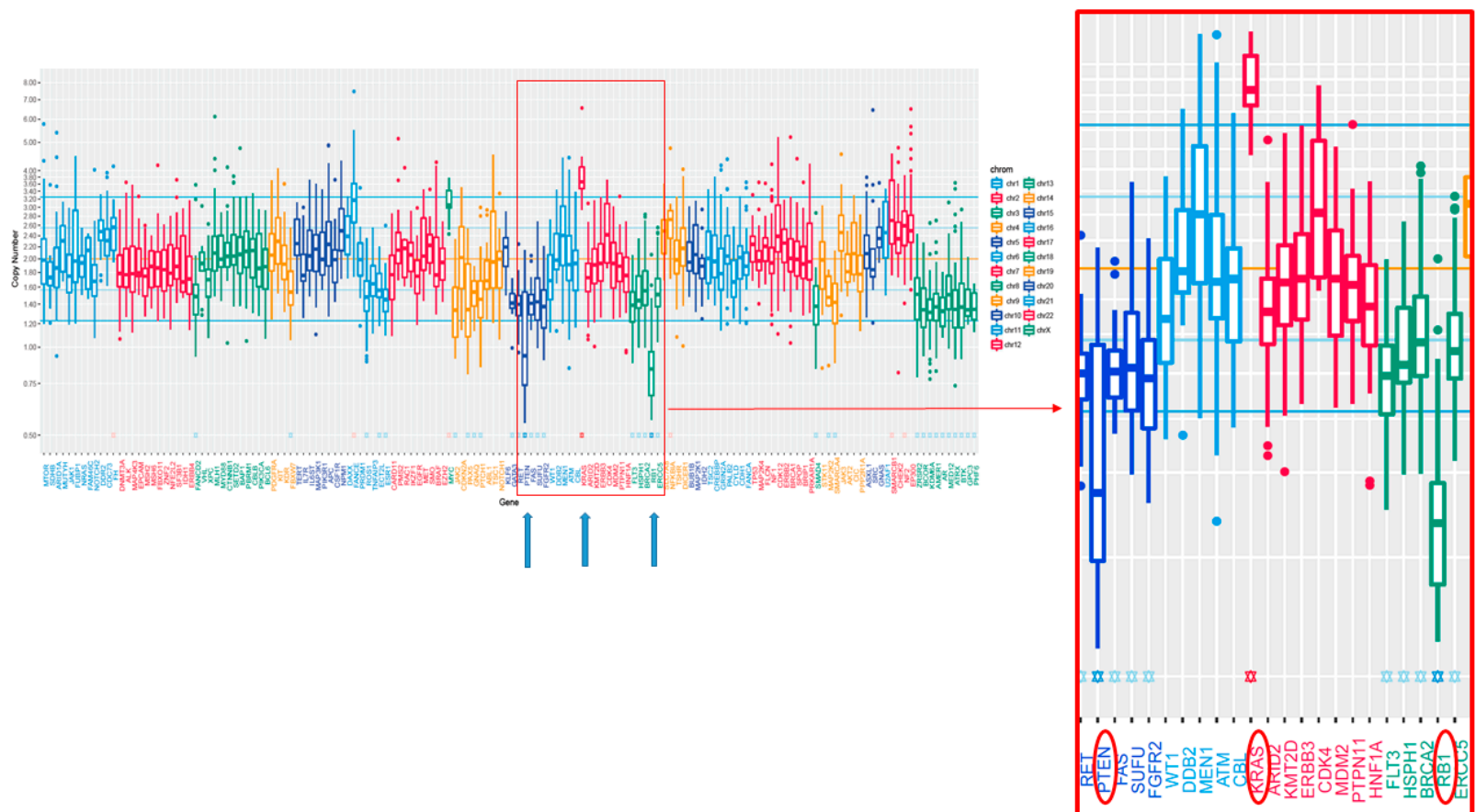

Figure 5. Copy number plot of cancer-related 160 genes. The horizontal axis indicates chromosomal location of the examined genes, and the vertical axis indicates the calculated copy number of each gene. The blue arrow indicated the amplification of KRAS and homodeletion of PTEN and RB1.

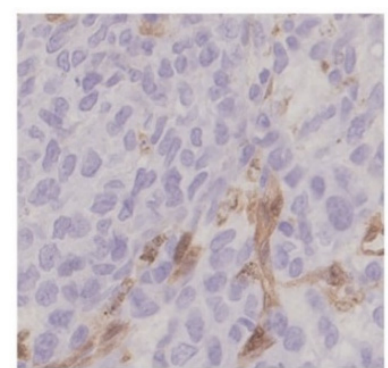

(a)

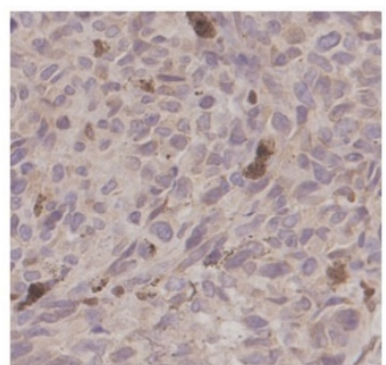

(b)

Figure 6. Immunohistochemical findings. Malignant melanoma was negative for PTEN (a: $20 \times)$ and RB1(b: $20 \times)$.

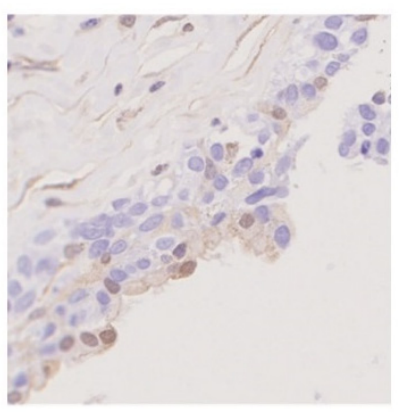

(a)

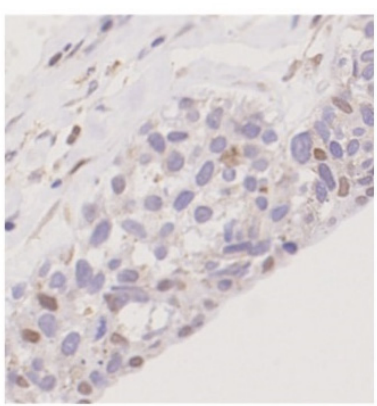

(b)

Figure 7. Immunohistochemical findings. The epithelium from mature cystic teratoma showed a mosaic pattern for PTEN (a: $10 \times)$ and RB1 (b: $10 \times)$. 


\section{Discussion and Conclusions}

MCTs constitute $10-20 \%$ of all ovarian neoplasms. They tend to be present in young women around the age of 30 years. MCTs are composed of well-differentiated derivations from at least two of the three germ cell layers. They contain developmentally mature skin, complete with hair follicles and sweat glands, and they occasionally include luxuriant clumps of long hair and pockets of sebum, blood, fat, bone, nails, teeth, eyes, cartilage, and thyroid tissue. MCTs are usually benign but undergo malignant transformation in less than $0.2 \%$ of cases, with an incidence of 1-3\% [15]. Several malignancies may develop from any of the three germ cell layers, such as adenocarcinoma, malignant thyroid struma, carcinoid tumors, melanomas, and a variety of soft tissue sarcomas [15]. The most common malignant evolution is squamous cell carcinoma from the ectoderm [15]. In a previous study, gene alterations of TP53, PIK3CA, and CDKN2A were frequently observed in squamous cell carcinoma derived from teratoma [16]. Meanwhile, MM-MCTs are extremely rare, with an estimated incidence of $<1 \%$; thus, the genomic characters are to be clarified.

We sequenced 160 cancer-related genes (Table S1) in the tumor sample obtained from the patient diagnosed with MM-MCT and accordingly detected KRAS amplification, as well as PTEN and RB1 HD, in the sample. Furthermore, an immunohistochemical analysis revealed that the tumor cells in the MCT area lost the expression of the PTEN and RB1 proteins. Based these observations, we hypothesized that the MCT harbored the same gene alteration in KRAS, PTEN, and RB1, and we speculated that the MM had arisen from the MCT. Additionally, we identified that the endometriosis area did not lose the expression of the PTEN and RB1 proteins, suggesting that endometriosis accidentally coexists with an MM-MCT.

In this case, we focused on the association between origin sites and genetic events. The origin site was an ovarian MCT, an uncommon origin site despite being observed most often in the cutaneous sites.

PTEN is a tumor suppressor gene that is mutated at a high frequency in a wide variety of human cancers (such as glioblastoma, prostate, breast, and osteosarcoma) and was recently identified as a key driver of osteosarcoma in a murine forward genetic screen $[17,18]$. PTEN encodes a lipid phosphatase that dephosphorylates phosphatidylinositol $(3,4,5)$ triphosphate (PIP3) to oppose the activity of PI3K, which functions as a PIP3 kinase and is constitutively activated and functions as an oncogene in many cancers [19]. The loss of PTEN causes the persistent activation of the Akt serine-threonine kinase, which promotes cell growth and proliferation, inhibits apoptosis, and controls metabolism by directly phosphorylating numerous downstream targets such as BAD and FOXO and transcription factors such as CASP9, MTOR, and MDM2 [20].

$R B 1$ is a tumor suppressor gene and is a key regulator of E2F transcription factors and cell cycle progression; thus, its deletion is often accompanied by defects in cell cycle exit and can result in an undifferentiated cellular phenotype [21-23]. In addition, pRB controls mesenchymal cell differentiation through interactions with RUNX2 to promote osteogenic differentiation [22] or by blocking PPAR- $\gamma$ expression to suppress adipogenesis [24]. Though less commonly associated with familial melanoma than with hereditary retinoblastoma, patients with the germline inactivation of RB1 are predisposed to melanoma [25]. However, RB1 HD has not been reported in PCM cases.

Regarding KRAS alterations, KRAS mutations or amplifications have not been described in human melanocytic lesions, whereas NRAS mutations have been correlated with the carcinogenesis of PCM.

In a previous study on patients with metastatic colorectal cancer, PTEN loss and KRAS mutations were shown to have beneficial effects on cetuximab and irinotecan treatment [26]; however, no treatment that was beneficial for all variants (PTEN/RB1/KRAS) was reported.

The recent and growing interest in the unique genetic pathways involved in neoplasia is partially a consequence of the promising developments in targeted therapies, which contribute to the increasing potential of personalized medicine strategies. Previous studies have identified a number of genetic abnormalities associated with oncogenes and tumor 
suppressor genes in the tumorigenesis of multiple cancer types. Based on the findings of this report, it is possible that PCM carcinogenesis differs from that of MM-MCT. Due to the lack of standard treatment for MM-MCT, our patient often underwent treatments based on immune checkpoint inhibitors, such as pembrolizumab. However, these treatments were not effective. In such cases of rare diseases, we have to consider targeted therapies based on the genetic profile in each case.

This is the first study to report the genomic profiling of MM-MCT. Further genetic analyses of MM-MCTs will undoubtedly help elucidate the mechanisms of the carcinogenesis of PCM and MM-MCTs, as well as provide a basis for novel gene-targeted therapy as a step toward the development of precision medicine strategies.

Supplementary Materials: The following are available online at https:/ / www.mdpi.com/1422-006 7/22/5/2436/s1, Table S1: Genes (160) examined in the PleSSision test.

Author Contributions: Conceptualization, H.N.; investigation, E.A., R.T.; resources, T.T., K.S., H.S.; data curation, S.T.; writing —original draft preparation, K.N.; writing—review and editing, E.A. and H.N. All authors have read and agreed to the published version of the manuscript.

Funding: This study was supported by the JSPS KAKENHI (Grant-in-Aid for Young Scientists B; grant 20K18232).

Institutional Review Board Statement: This study was approved by the Institutional Review Board (IRB) of Kumagaya General Hospital (approval number: 44).

Informed Consent Statement: Informed consent was obtained from the subject involved in the study.

Data Availability Statement: The data presented in this study are available on request from the corresponding author.

Conflicts of Interest: The authors declare no conflict of interest.

\author{
Abbreviations \\ HD Homozygous deletion \\ PCM Primary cutaneous melanoma \\ MCT Mature cystic teratoma \\ MM-MCT Malignant melanoma arising from ovarian mature cystic teratoma
}

\title{
References
}

1. Zhang, T.; Dutton-Regester, K.; Brown, K.M.; Hayward, N.K. The genomic landscape of cutaneous melanoma. Pigment Cell Melanoma Res. 2016, 29, 266-283. [CrossRef] [PubMed]

2. Shain, A.H.; Yeh, I.; Kovalyshyn, I.; Sriharan, A.; Talevich, E.; Gagnon, A.; Dummer, R.; North, J.; Pincus, L.; Ruben, B.; et al. The genetic evolution of melanoma from precursor lesions. N. Engl. J. Med. 2015, 373, 1926-1936. [CrossRef] [PubMed]

3. Wong, S.Q.; Behren, A.; Mar, V.J.; Woods, K.; Li, J.; Martin, C.; Sheppard, K.E.; Wolfe, R.; Kelly, J.; Cebon, J.; et al. Whole exome sequencing identifies a recurrent RQCD1 P131L mutation in cutaneous melanoma. Oncotarget 2015, 6, 1115-1127. [CrossRef] [PubMed]

4. Shain, A.H.; Garrido, M.; Botton, T.; Talevich, E.; Yeh, I.; Sanborn, J.Z.; Chung, J.; Wang, N.J.; Kakavand, H.; Mann, G.J.; et al. Exome sequencing of desmoplastic melanoma identifies recurrent NFKBIE promoter mutations and diverse activating mutations in the MAPK pathway. Nat. Genet. 2015, 47, 1194-1199. [CrossRef] [PubMed]

5. Krauthammer, M.; Kong, Y.; Bacchiocchi, A.; Evans, P.; Pornputtapong, N.; Wu, C.; McCusker, J.P.; Ma, S.; Cheng, E.; Straub, R.; et al. Exome sequencing identifies recurrent mutations in NF1 and RASopathy genes in sun-exposed melanomas. Nat. Genet. 2015, 47, 996-1002. [CrossRef] [PubMed]

6. Arafeh, R.; Qutob, N.; Emmanuel, R.; Keren-Paz, A.; Madore, J.; Elkahloun, A.; Wilmott, J.S.; Gartner, J.J.; Di Pizio, A.; WinogradKatz, S.; et al. Recurrent inactivating RASA2 mutations in melanoma. Nat. Genet. 2015, 47, 1408-1410. [CrossRef]

7. Andrews, H.R. Primary melanotic sarcoma of the ovary. Trans. Obstet. Soc. 1901, 43, 228-231.

8. Davis, G.L. Malignant melanoma arising in mature ovarian cystic teratoma (dermoid cyst). Report of two cases and literature analysis. Int. J. Gynecol. Pathol. 1996, 15, 356-362. [CrossRef]

9. Moehrle, M.; Fischbach, H.; Nuessle, B.; Rassner, G. Primary malignant melanoma arising in a cystic necrotic ovarian teratoma. Eur. J Obstet. Gynecol. Reprod. Biol. 2001, 99, 268-271. [CrossRef] 
10. Sarnie, K.N.; Haddad, A.; Fortune, D. 31. A malignant melanoma arising in a mature cystic teratoma. Pathology 2014, 46 , S116. [CrossRef]

11. Ross, J.S.; Wang, K.; Rand, J.V.; Sheehan, C.E.; Jennings, T.A.; Al-Rohil, R.N.; Otto, G.; Curran, J.C.; Palmer, G.; Downing, S.; et al. Comprehensive genomic profiling of relapsed and metastatic adenoid cystic carcinomas by next-generation sequencing reveals potential new routes to targeted therapies. Am. J. Surg. Pathol. 2014, 38, 235-238. [CrossRef] [PubMed]

12. Nakamura, K.; Aimono, E.; Tanishima, S.; Imai, M.; Nagatsuma, A.K.; Hayashi, H.; Yoshimura, Y.; Nakayama, K.; Kyo, S.; Nishihara, H. Olaparib monotherapy for BRIP1-mutated high-grade serous endometrial cancer. JCO Precis. Oncol. 2020, 4, PO.19.00368. [CrossRef] [PubMed]

13. Nakamura, K.; Aimono, E.; Tanishima, S.; Nomura, H.; Imai, M.; Hayashi, H.; Nishihara, H. Genetic profiling of patients with adenoid cystic carcinoma of the Bartholin's glands reveals potential new routes for targeted therapies: A case report. Diagn. Pathol. 2020, 15, 64. [CrossRef]

14. Nakamura, K.; Aimono, E.; Tanishima, S.; Imai, M.; Nagatsuma, A.K.; Hayashi, H.; Yoshimura, Y.; Nakayama, K.; Kyo, S.; Nishihara, H. Intratumoral genomic heterogeneity may hinder precision medicine strategies in patients with serous ovarian carcinoma. Diagnostics 2020, 10, 200. [CrossRef]

15. Rim, S.; Kim, S.; Choi, H. Malignant transformation of ovarian mature cystic teratoma. Int. J. Gynecol. Cancer 2006, 16, 140-144. [CrossRef]

16. Cooke, A.L.; Ennis, D.; Evers, L.; Dowson, S.; Chan, M.Y.; Paul, J.; Hirschowitz, L.; Glasspool, R.M.; Singh, N.; Bell, S.; et al. The driver mutational landscape of ovarian squamous cell carcinomas arising in mature cystic teratoma. Clin. Cancer Res. 2017, 23, 7633-7640. [CrossRef] [PubMed]

17. Li, J.; Yen, C.; Liaw, D.; Podsypanina, K.; Bose, S.; Wang, S.I.; Puc, J.; Miliaresis, C.; Rodgers, L.; McCombie, R.; et al. PTEN, a putative protein tyrosine phosphatase gene mutated in human brain, breast, and prostate cancer. Science 1997, 275, $1943-1947$. [CrossRef] [PubMed]

18. Stambolic, V.; Suzuki, A.; de la Pompa, J.L.; Brothers, G.M.; Mirtsos, C.; Sasaki, T.; Ruland, J.; Penninger, J.M.; Siderovski, D.P.; Mak, T.W. Negative regulation of PKB/Akt-dependent cell survival by the tumor suppressor PTEN. Cell 1998, 95, 29-39. [CrossRef]

19. Cantley, L. The phosphoinositide 3-kinase pathway. Science 2002, 296, 1655-1657. [CrossRef] [PubMed]

20. Manning, B.D.; Cantley, L.C. AKT/PKB signaling: Navigating downstream. Cell 2007, 129, 1261-1274. [CrossRef]

21. Dyson, N. The regulation of E2F by pRB-family proteins. Genes Dev. 1998, 12, 2245-2262. [CrossRef]

22. Gutierrez, G.M.; Kong, E.; Sabbagh, Y.; Brown, N.E.; Lee, J.S.; Demay, M.B.; Thomas, D.M.; Hinds, P.W. Impaired bone development and increased mesenchymal progenitor cells in calvaria of RB1-/- mice. Proc. Natl. Acad. Sci. USA 2008, 105, 18402-18407. [CrossRef] [PubMed]

23. Calo, E.; Quintero-Estades, J.A.; Danielian, P.S.; Nedelcu, S.; Berman, S.D.; Lees, J.A. Rb regulates fate choice and lineage commitment in vivo. Nature 2010, 466, 1110-1114. [CrossRef] [PubMed]

24. Fajas, L.; Landsberg, R.L.; Huss-Garcia, Y.; Sardet, C.; Lees, J.A.; Auwerx, J. E2Fs regulate adipocyte differentiation. Dev. Cell 2002, 3, 39-49. [CrossRef]

25. Draper, G.J.; Sanders, B.M.; Kingston, J.E. Second primary neoplasms in patients with retinoblastoma. Br. J. Cancer 1986, 53, 661-671. [CrossRef] [PubMed]

26. Loupakis, F.; Pollina, L.; Stasi, I.; Ruzzo, A.; Scartozzi, M.; Santini, S.; Masi, G.; Graziano, F.; Cremolini, C.; Rulli, E.; et al. PTEN expression and KRAS mutations on primary tumors and metastases in the prediction of benefit from cetuximab plus irinotecan for patients with metastatic colorectal cancer. J. Clin. Oncol. 2009, 27, 2662-2669. [CrossRef] [PubMed] 